\title{
Knowledge translation: an overview and recommendations in relation to the Fourth Canadian Consensus Conference on the Diagnosis and Treatment of Dementia
}

\author{
Cheryl Cook ${ }^{1,2}$ and Kenneth Rockwood*1,2,3
}

\begin{abstract}
The growing population of persons with dementia in Canada and the provision of quality care for this population is an issue that no healthcare authority will escape. Physicians often view dementia as a difficult and time-consuming condition to diagnose and manage. Current evidence must be effectively transformed into usable recommendations for physicians; however, we know that use of evidencebased practice recommendations is a challenge in all realms of medical care, and failure to utilize these leads to less than optimal care for patients. Despite this expanding need for readily available resources, knowledge translation (KT) is often seen as a daunting, if not confusing, undertaking for researchers. Here we offer a brief introduction to the processes around $K T$, including terms and definitions, and outline some common KT frameworks including the knowledge to action cycle, the Promoting Action on Research Implementation in Health Services framework and the Consolidated Framework for Implementation Research. We also outline practical steps for planning and executing a KT strategy particularly around the implementation of recommendations for practice, and offer recommendations for $\mathrm{KT}$ planning in relation to the Fourth Canadian Consensus Conference on the Diagnosis and Treatment of Dementia.
\end{abstract}

*Correspondence: Kenneth.Rockwood@dal.ca

'Division of Geriatric Medicine, Dalhousie University, 5955 Veterans' Memorial Lane, Room 2650, Halifax, NS B3H 2E1, Canada

Full list of author information is available at the end of the article

\section{Introduction}

The growing population of persons with dementia in Canada and the provision of quality care for this population is an issue that no healthcare authority will escape. Physicians often view dementia as a difficult and timeconsuming condition to diagnose and manage [1-3]. Current evidence must be effectively transformed into usable recommendations for physicians; however, we know that use of evidence-based practice recommendations is a challenge in all realms of medical care, and failure to utilize these leads to less than optimal care for patients [4-7]. This is no different in dementia care, where physicians often perceive a lack of recommendations even when these exist [8]. While continuing professional development (CPD) and continuing medical education (CME) have traditionally attempted to address this need for effective implementation of recommendations, increasingly it is knowledge translation (KT) - with its focus on health outcomes, interdisciplinary approach, and broad outlook which encompasses and expands on many of the concepts of CPD and CME - that is being called upon to improve the use of evidence in practice [5].

Despite this growing emphasis, KT often appears on the surface to be a daunting topic. Over 90 terms have been coined to describe KT $[9,10]$ and there are a variety of theories and implementation frameworks for an individual researcher or group to choose from when considering the use of KT.

Here we offer a brief introduction to some KT frameworks, outline practical steps for planning and executing a KT strategy around the implementation of recommendations for practice, and offer recommendations for KT planning in relation to the Fourth Canadian Consensus Conference on the Diagnosis and Treatment of Dementia (CCCDTD4).

\section{Defining knowledge translation}

As may be expected for any concept with dozens of terms available to describe it, there are a variety of 
working definitions for KT. The Canadian Institutes for Health Research define KT as '... a dynamic and iterative process that includes synthesis, dissemination, exchange and ethically-sound application of knowledge to improve the health of Canadians, provide more effective health services and products and strengthen the health care system' [11]. The National Center for the Dissemination of Disability Research (USA) describes it for their purposes as the collaborative and systematic review, assessment, identification, aggregation, and practical application of high-quality disability and rehabilitation research by key stakeholders (i.e., consumers, researchers, practitioners, and policymakers) for the purpose of improving the lives of individuals with disabilities' [12]. Despite the many definitions, the common thread is '... a move beyond the simple dissemination of knowledge into the actual use of knowledge' [10].

KT should not be used synonymously with dissemination, just as it should not be confused with commercialization, technology transfer, or even CME. In all cases, it takes a broader view with additional focus on the quality of the evidence being used, the involvement of end-users, the methods for transferring the knowledge to these end-users, and the evaluation of the impact of the implementation $[10,13]$.

\section{Frameworks for knowledge translation}

The need for organized processes and checks of barriers and facilitators in the translation of knowledge demands a framework on which one can build, and with which testable and useful interventions can proceed in a measured, thoughtful way. Here we will describe three common frameworks for KT.

\section{Knowledge to action}

The knowledge to action (KTA) framework, proposed by Graham and colleagues as a framework for the transfer of research findings into practice, is presented as two concepts: knowledge creation and the action cycle [13] (Figure 1). In practice, the two concepts are fluid and do not always occur exclusive of each other.

Knowledge creation is represented in the diagram by a central funnel. As it moves down the funnel, knowledge becomes more refined through the steps of inquiry (for example, primary research), synthesis (for example, systematic review) and creation of tools or products (for example, recommendations).

The action cycle moves from this process of knowledge refinement into the implementation of the knowledge. There are several steps in the action cycle, derived from theories of planned action [13], all of which may inform each other, often resulting in a nonsequential cycle. The steps are as follows:
1. Identifying the problem as well as the knowledge to address this (as well as addressing the usefulness and validity of the knowledge).

2. Adapting the knowledge to the local context by assessing the value and usefulness of the knowledge to the setting for which it is intended.

3. Assessing barriers and facilitators related to the knowledge to be adopted, the potential adopters, and the context in which the knowledge will be used.

4. Developing and executing the knowledge to action plan and any strategies to promote awareness and use of the knowledge.

5. Monitoring the use of the knowledge to determine the effectiveness of the plan, as well as implementing any required changes indicated by this. If at this stage knowledge use is not at the desired or predicted level, a reassessment may occur of the known barriers to adoption - for example, have new barriers occurred since implementation? - the adopters' outlook, and so forth.

6. Evaluating the impact of the knowledge use to determine whether it has effected the desired outcomes, as well as the success or worth of the KT plan.

7. Sustaining the use of the knowledge over time. Barriers to ongoing use of the knowledge may not be the same as those for implementation of it, so this is considered a separate phase.

An important component to each piece of this (or any) framework is the consideration of (and involvement with) the target audience to understand how/if they can use of the knowledge, and the context within which they exist [14].

\section{Promoting Action on Research Implementation in Health Service framework}

The Promoting Action on Research Implementation in Health Service (PARiHS) framework, developed by Kitson and colleagues [15-18], focuses on the importance of the context or environment in which a change is implemented, the level and type of evidence being translated, the method of facilitation for this, and the relationship between these three. While it is considered a useful and highly practical framework, PARiHS remains largely untested [19]. The framework considers the attributes of evidence, context and facilitation as well as the overall high to low attributes for each of the three. They argue that implementation works best when there is robust scientific evidence, an environment that is welcoming to this evidence, and skilled facilitation to assist with the implementation. Recent work has described a further evaluation of this model that highlights the need for a two-stage process, concentrating first on the evidence and contexts, and utilizing data from this process to better inform the method of facilitation [19]. 


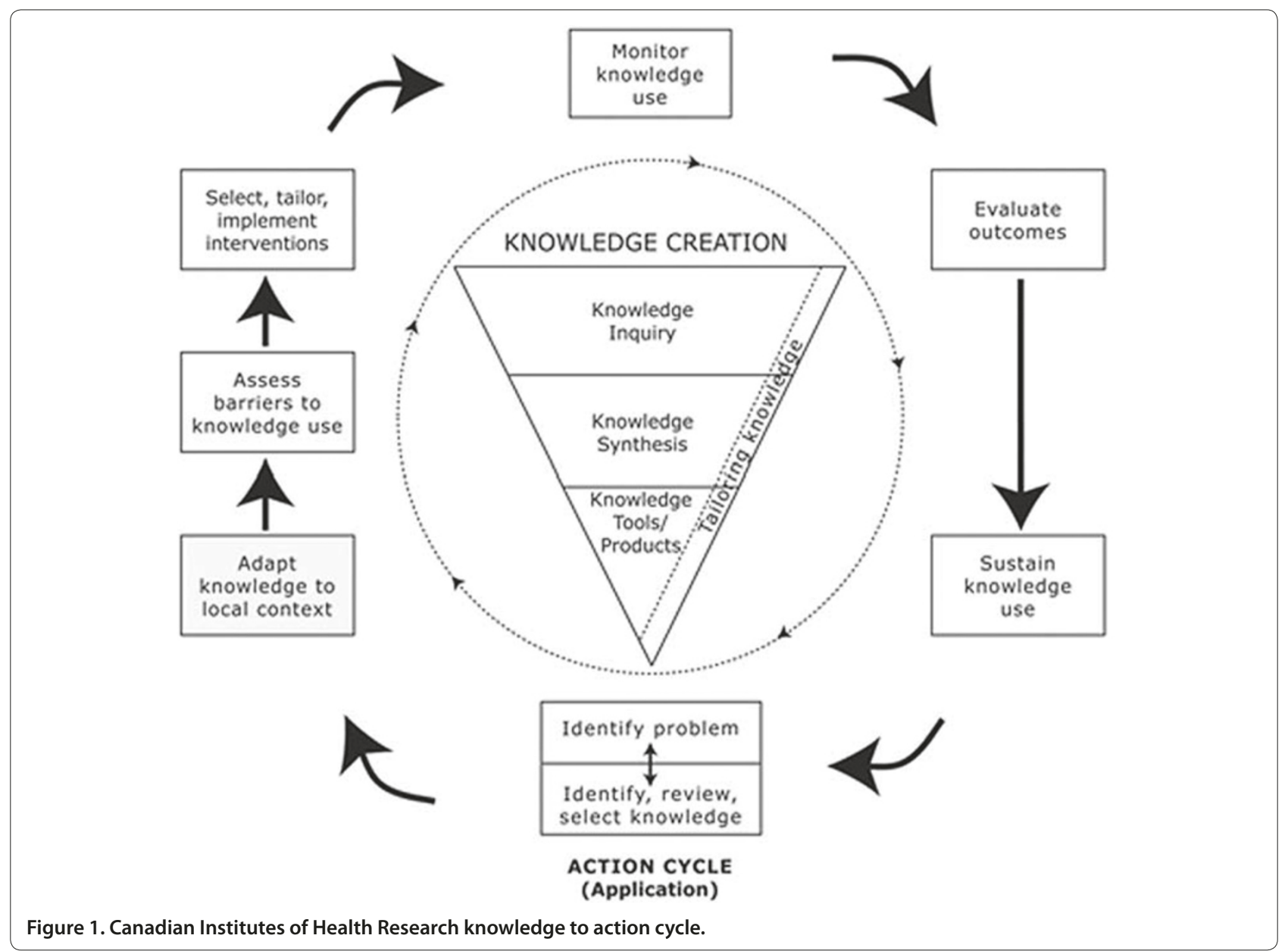

\section{Consolidated Framework for Implementation Research}

Developed by a group in the US Veterans Health Administration, the Consolidated Framework for Implementation Research (CFIR) represents the consolidation of common constructs derived from a review of existing theories of knowledge transfer [20]. The CFIR can be used to assist identification of barriers and facilitators to interventions, to track implementation processes, and to explore the factors that influence implementation and how implementation influences interventions.

The CFIR outlines five domains and the common constructs for each of these [21]:

1. Intervention characteristics look at the intervention's complexity, source, strength and quality of evidence, relative advantage, ability to be trialed, quality of design and packaging, and cost.

2. The outer setting includes the patient's need and resources, cosmopolitanism (the degree to which a group or organization is networked with other organizations), peer pressure and any incentives or external policies that could affect implementation.
3. The inner setting considers structural contexts of an organization, the nature and quality of social networks and formal/informal communications within an organization, the culture of a given setting in terms of its norms, values and basic assumptions, and readiness for implementation.

4. The characteristics of individuals are constructs that include the knowledge and beliefs held by individuals toward the intervention, self-efficacy (individual belief in the capacity to achieve the goals of the implementation), the individual state of change (the phase an individual is in during a given point of progress toward sustained use of the intervention), the individual identification with the organization, and other personal attributes such as motivation, values, competence, and so forth.

5. The process considers the constructs of planning, engaging appropriate individuals (for example, opinion leaders), executing the implementation, and reflecting and evaluating.

While the CFIR is relatively new, it is considered a useful tool not only for understanding implementation 
itself, but also for ensuring more effective implementations [20].

\section{Knowledge translation planning}

Planning a KT strategy, regardless of the definition and framework used, benefits from guiding questions that allow organization of this process. Lavis and colleagues offer five questions for KT planning that ask [22]:

1 . What is the message or knowledge to be transferred?

2. To whom should it be transferred?

3. By whom should it be transferred?

4. How should it be transferred?

5 . What is the desired effect or impact?

These five questions inform each other, so this is rarely a linear process. As consideration is given to one area, it may require adjustments in others.

\section{What is the message or knowledge to be transferred?}

The amount of evidence available to physicians has increased dramatically in recent years [23] and many evidence-based recommendations have been developed that aim to improve patient care. This explosion of available information means that scrutiny of the quality of evidence being translated at the outset of this process is crucial. Translating knowledge from a body of work rather than a single study is considered preferable [22], and while results from rigorous research are considered the foundation of good KT, there is a wide variety and scope of evidence that can and should be considered in many projects. Included in this can be everything from contextual information pertinent to an intervention (for example, resource availability, constraints in a given context) to that traditionally considered of higher quality such as clinical trials or systematic reviews [24,25]. The inclusion of evidence from a variety of sources logically leads to a process of evaluating the strengths and limitations of each type. Additionally, your message should be crafted bearing in mind the potential audience, such as primary care physicians, and consideration should be given to how and in what format that audience prefers to receive information, and what the evidence says about the effectiveness of the methods selected.

\section{To whom should it be transferred?}

Determining the target audience(s) for translation informs a range of others areas in $\mathrm{KT}$ planning. Inclusion of members of the target audience in the $\mathrm{KT}$ processes allows for better understanding of potential barriers, facilitators, and needs of the particular group. Active involvement of practitioners in the translation of recommendations, particularly face to face, is recognized as the most efficient way to create a strategy that is likely to have its intended impact [26-28]. While it is important to include a wide variety of stakeholders in this process, there must still be consideration given to the appropriateness of a given audience based on your message, desired outcomes, and so forth. If you are translating evidence into practice recommendations, is your target group able to act on these? If, for example, a policy change is required, then this additional audience must be considered along with their differing preferences and requirements. Readiness for change is another important factor. Individuals often work within organizations, and the culture and values of this organization may impact the individuals' readiness to accept new information or recommendations. While the cultivation of such relationships is not necessarily a skill many researchers have considered, it is indentified as a key element in effective dissemination [29].

\section{By whom should it be transferred?}

Consideration should be given to how the target audience will view the credibility of the messenger. This may be judged on multiple factors including the evidence being translated, but especially the individual or organization providing this. The use of opinion leaders is assumed to lend credibility with a particular audience, and drawing on respected physician organizations/colleagues has been shown to be effective for adoption of clinical recommendations [22]. The inclusion of members from your target audience in your KT planning will assist you in understanding which messengers may be most suitable for your intended audience.

\section{How should it be transferred?}

There are a wide variety of methods to choose from when considering dissemination, all of which will be influenced by your message, your intended impact and, very importantly, the audience(s) you wish to influence. Lomas proposes a three-part taxonomy for considering the different levels of dissemination (and their usefulness): diffusion, dissemination, and implementation [30].

Diffusion includes many passive types of information dissemination with which academics will be quite familiar, such as publication in academic journals or presentation of findings at a conference. In terms of uptake by your intended audience, this is considered fairly unplanned and uncontrolled [31].

Dissemination represents activities that often come to mind when people talk about $\mathrm{KT}$, and are tailored to the specific target audience. These may include less active forms of dissemination, such as translating your results into brochures or policy briefs, or more active forms such as small group sessions to disseminate findings or the use of a knowledge-broker or network [31].

Implementation takes a further step, looking to specifically address and overcome barriers to uptake in order to encourage adoption. Use of frameworks such as 
the Clinical Practice Recommendations Framework for Improvement can serve as a useful guide for identification of barriers and facilitators to uptake, allowing for specific tailoring of the KT plan to tackle these [14].

A more comprehensive list of options is available through Barwick's Knowledge Translation Planning Template- $\mathrm{R}^{\mathrm{TM}}$ [32]. This template ranks dissemination activities or tools from mostly effective (for example, educational outreach, combined interventions) to unknown effects (for example, arts-based KT, social media).

\section{With what effect or impact?}

What effect or change do you seek from your audience? In what realm do you wish to see an impact? How you will evaluate this? Barwick's Knowledge Translation Planning Template- $\mathrm{R}^{\mathrm{sm}}$ again offers a comprehensive set of options for reflection and goal planning for your audience (for example, behavior change, practice change, imparting of new tools, and so forth) as well as the realm in which you wish to have an impact (for example, patient outcomes, policy, research) [32]. The considerations here will be heavily influenced by which groups you have chosen as your target audience(s) (for example, primary care physicians) as well as the knowledge to be translated (for example, practice recommendations), and will influence your choices for method of transfer.

As $\mathrm{KT}$ is a process that takes considerable time and resources, evaluation of these is critical [14]. Evaluation should be tailored to match the audience and the desired outcomes, thus indicators to be considered can include assessments of reach, usefulness, use, partnerships, practice change, program/services and policy [32]. Methods for evaluation can be both qualitative and quantitative (or mixed) but should be explicit and valid, as well as realistic and appropriate for the given target audience, setting and desired outcomes [14].

\section{Considerations for CCCDTD4}

Our proposal suggests an approach that uses the Canadian Institutes of Health Research KTA model [13]. While all three KT frameworks presented here are useful and robust, KTA is a widely used model with which the Canadian Dementia Knowledge Translation Network (CDKTN) has worked previously. As the knowledge creation cycle is primarily complete (with the CCCDTD4 recommendations) the focus would be the on the action cycle.

In identifying the problem, consideration should be given to the fact that dementia is perceived by physicians as a difficult and time-consuming condition to treat and diagnose [1] and evidence-based clinical practice recommendations are often underused [8]. Thus it is crucial that the most current evidence-based clinical practice recommendations are effectively translated to assist physicians in providing the best care possible for patients with dementia.

Adapting the recommendations to the local context and intended target audience (that is, primary care physicians and their teams) and understanding potential barriers to uptake of these new recommendations can be greatly assisted through the inclusion of various stakeholder groups in the planning and execution process. Along with an examination of the current literature around dissemination to the intended target audience, there are a variety of options that (dependent on the planning structure for the project) include the following: inviting knowledge user groups/individuals to participate in a project committee or other project activities; surveying primary care health providers about their preferences for receiving such information; and participating in events or activities aimed at the target audience of primary care with the goal of engaging them in discussions around preferred methods of dissemination for such information.

In considering the development and execution of the KT plan, we would recommend an approach that targets not only the physicians, but also their healthcare teams. For that reason we would initially suggest the following be considered:

1. An online learning module (for example, CME) for the new recommendations that is aimed at a primary care audience - content for this can be derived from the CCCDTD4 and modified by the CDKTN.

2. The CDKTN's knowledge exchange arm (Canadian Dementia Resource and Knowledge Exchange (CDRAKE)) can provide up to three webinars based on the recommendations, aimed at a broader audience that includes a variety of nonphysician healthcare professions.

3. Slide decks based on the new (and previous) Canadian Consensus Conference on the Diagnosis and Treatment of Dementia recommendations aimed at all treating physicians will be made available for download and use via the CDKTN's website.

4. Other existing resources aimed at assisting primary care physicians with diagnosis and treatment of dementia can be considered for inclusion of the new recommendations. The CDKTN can work with interested groups to assist with these updates, and to ensure promotion of these tools and services to a wide audience.

We also recommend that efforts are made to widely promote the availability of these new recommendations and the tools mentioned above. Some options available to accomplish this are as follows:

1. Engagement of key opinion leaders (via, for example, CDKTN's networks) in primary care and dementia 
care as champions for the promotion of the dissemination. Promotional booths at events such as The Family Medicine Forum (4000 to 5000 family physicians from across Canada) and annual meetings of the Canadian Geriatrics Society and the Canadian Academy of Geriatric Psychiatry. These events allow in-person promotion of the new recommendations and all online tools to both users and to teachers.

2. Promotion via the Primary Care Community of Practice (CDRAKE)

3. Social media channels: CDKTN and CDRAKE's established Twitter and Facebook feeds are connected to over 2000 people and organizations in healthcare. The CDKTN also has an active website and member newsletter that is distributed bimonthly to over 700 members.

With regard to monitoring the use of the knowledge and evaluating the success of the KT plan, there are also a variety of options. The online platform chosen for the CME should allow for evaluation of a variety of factors including number of participants, demographics of participants, as well as post-CME evaluation (via email) of content and impact on practice, to allow for refinement of this resource. The CDRAKE webinars should also be similarly evaluated. Preparation of a formal evaluation plan that considers reach, impact and practice change should be expected.

In conclusion, the recommendation offered here is the use of the Canadian Institutes of Health Research KTA framework to allow for structured dissemination and evaluation of the new CCCDTD4 recommendations. We recommend the use of Lavis' five questions as a commonsense checklist for the $\mathrm{KT}$ planning and execution process. Preparation of a formal evaluation plan that considers reach, impact and practice change should also be included.

\section{Abbreviations}

CCCDTD4, Fourth Canadian Consensus Conference on the Diagnosis and Treatment of Dementia; CDKTN, Canadian Dementia Knowledge Translation Network; CDRAKE, Canadian Dementia Resource and Knowledge Exchange; CFIR, Consolidated Framework for Implementation Research; CME, continuing medical education; CPD, continuing professional development; KT, knowledge translation; KTA, knowledge to action; PARiHS, Promoting Action on Research Implementation in Health Services.

\section{Competing interests}

The authors declare that they have no competing interests.

\section{Acknowledgements}

The authors would like to thank the Canadian Dementia Knowledge Translation Network whose funding has allowed the authors to work on this review.

\section{Declarations}

This article has been published as part of Alzheimer's Research \& Therapy Volume 5 Supplement 1, 2013: Background documents to the 4th Canadian Consensus Conference on the Diagnosis and Treatment of Dementia (CCCDTD4). The full contents of the supplement are available online at http://alzres.com/supplements/5/S1.
Publication charges for the supplement were funded by the Canadian Consensus Conference on the Diagnosis and Treatment of Dementia (CCCDTD). Although residual conference funds used include contributions from pharmaceutical companies, no commercial organization has been involved in the selection of participants, choice of topics, preparation of background papers or recommendations. In kind support was also provided by the Canadian Dementia Knowledge Translation Network, and the offices of Drs Serge Gauthier (McGill University), Christopher Patterson (McMaster University) and Howard Chertkow (McGill University), whose role as Guest Editors involved the coordination of the project without involvement in the journal's standard peer review process which applied for all articles.

\section{Author details}

'Canadian Dementia Knowledge Translation Network, 5955 Veterans' Memorial Lane, Room 1427, Halifax, NS B3H 2E1, Canada. 'Geriatric Medicine Research, 5955 Veterans' Memorial Lane, Room 1421, Halifax, NS B3H 2E1, Canada.

${ }^{3}$ Division of Geriatric Medicine, Dalhousie University, 5955 Veterans' Memorial Lane, Room 2650, Halifax, NS B3H 2E1, Canada.

Published: 8 July 2013

\section{References}

1. Pimlott NJ, Persaud M, Drummond N, Cohen CA, Silvius JL, Seigel K, Hollingworth GR, Dalziel WB: Family physicians and dementia in Canada: Part 2. Understanding the challenges of dementia care. Can Fam Physician 2009, 55:508-509.e1-e7.

2. Hinton L, Franz CE, Reddy G, Flores Y, Kravitz RL, Barker JC: Practice constraints, behavioral problems, and dementia care: primary care physicians' perspectives. J Gen Intern Med 2007, 22:1487-1492.

3. Harris DP, Chodosh J, Vassar SD, Vickrey BG, Shapiro MF: Primary care providers' views of challenges and rewards of dementia care relative to other conditions. J Am Geriatr Soc 2009, 57:2209-2216.

4. Straus SE, Tetroe JM, Graham ID: Knowledge translation is the use of knowledge in health care decision making. J Clin Epidemiol 2011, 64:6-10.

5. Davis D, Davis N: Selecting educational interventions for knowledge translation. CMAJ 2003, 182:89-93

6. Green LA, Seifert CM: Translation of research into practice: why we can't 'just do it'. J Am Board Fam Pract 2005, 18:541-545.

7. Westfall JM, Mold J, Fagnan L: Practice-based research - 'Blue Highways' on the NIH roadmap. JAMA 2007, 297:403-406.

8. Pimlott NJ, Persaud M, Drummond N, Cohen CA, Silvius JL, Seigel K, Hollingworth GR, Dalziel WB: Family physicians and dementia in Canada: Part 1. Clinical practice guidelines: awareness, attitudes, and opinions. Can Fam Physician 2009, 55:506-507.e1-e5.

9. McKibbon KA, Lokker C, Wilczynski NL, Ciliska D, Dobbins M, Davis DA, Haynes RB, Straus SE: A cross-sectional study of the number and frequency of terms used to refer to knowledge translation in a body of health literature in 2006: a Tower of Babel? Implement Sci 2010, 5:16.

10. Straus S, Tetroe J, Graham I: Defining knowledge translation. Can Med Assoc J 2009, 181:3-4.

11. Canadian Institutes for Health Research: About Knowledge Translation [http://www.cihr.ca/e/29418.html]

12. National Center for the Dissemination of Disability Research: Knowledge Translation [http://www.ncddr.org/knowledge_trans_over.html]

13. Graham ID, Logan J, Harrison MB, Straus SE, Tetroe J, Caswell W, Robinson N: Lost in knowledge translation: time for a map? J Contin Educ Health Prof 2006, 26:13-24.

14. Straus S, Tetroe J, Graham I (Eds): Knowledge Translation in Health Care: Moving from Evidence to Practice. West Sussex, UK: Blackwell Publishing; 2009.

15. Kitson A, Harvey G, McCormack B: Enabling the implementation of evidence based practice: a conceptual framework. Qual Health Care 1998, 7:149-158.

16. Harvey G, Loftus-Hills A, Rycroft-Malone J, Titchen A, Kitson A, McCormack B, Seers K: Getting evidence into practice: the role and function of facilitation. J Adv Nurs 2002, 37:577-588.

17. Rycroft-Malone J: The PARiHS framework - a framework for guiding the implementation of evidence-based practice. J Nurs Care Qual 2004, 19:297-304.

18. McCormack B, Kitson A, Harvey G, Rycroft-Malone J, Titchen A, Seers K: Getting evidence into practice: the meaning of 'context'. J Adv Nurs 2002, 38:94-104. 
19. Kitson AL, Rycroft-Malone J, Harvey G, McCormack B, Seers K, Titchen A: Evaluating the successful implementation of evidence into practice using the PARiHS framework: theoretical and practical challenges. Implement Sci 2008, 3:1.

20. Damschroder L, Aron D, Keith R, Kirsch S, Alexander J, Lowery J: Fostering implementation of health services research findings into practice: a consolidated framework for advancing implementation science. Implement Sci 2009, 4:50.

21. US Department of Vaterans' Affairs: CFIR Wiki [http://wiki.cfirwiki.net/index.php?title=Main_Page]

22. Lavis JN, Robertson D, Woodside JM, McLeod CB, Abelson J; Knowledge Transfer Study Group: How can research organizations more effectively transfer research knowledge to decision makers? Milbank Q 2003, 81:221248, 171-172

23. Gass D: Can family physicians practise good medicine without following clinical practice guidelines? No. Can Fam Physician 2010, 56:519-521, 523525; discussion e206-e207.

24. Glasgow RE, Emmons KM: How can we increase translation of research into practice? Types of evidence needed. Annu Rev Public Health 2007, 28:413-433.

25. Rycroft-Malone J, Seers K, Titchen A, Harvey G, Kitson A, McCormack B: What counts as evidence in evidence-based practice? J Adv Nurs 2004, 47:81-90.

26. Lomas J: Using 'linkage and exchange' to move research into policy at a Canadian foundation. Health Aff (Millwood) 2000, 19:236-240.
27. Harrison M, Legare F, Graham I, Fevers B: Adapting clinical practice guidelines to local context and assessing barriers to their use. Can Med Assoc J 2010, 182:78-84

28. Rynes S, Bartenuk J, Daft R: Across the great divide: knowledge creation and transfer between practitioners and academics. Acad Manage J 2010, 44:340-355.

29. Barwick M, Boydell K: A Pragmatic Review of Knowledge Translation: Moving Forward in Cardiovascular Disease and Hypertension. Toronto, ON: Heart and Stroke Foundation of Ontario; 2007.

30. Lomas J: Diffusion, dissemination, and implementation: who should do what? Ann N Y Acad Sci 1993, 703:226-235; discussion 235-237.

31. Gagnon ML: Moving knowledge to action through dissemination and exchange. J Clin Epidemio/ 2011, 64:25-31.

32. Barwick M: Knowledge Translation Planning Template-R [http://www.melaniebarwick.com/training.php]

doi:10.1186/alzrt202

Cite this article as: Cook C, Rockwood K: Knowledge translation: an overview and recommendations in relation to the Fourth Canadian Consensus Conference on the Diagnosis and Treatment of Dementia. Alzheimer's Research \& Therapy 2013, 5(Suppl 1):S6. 